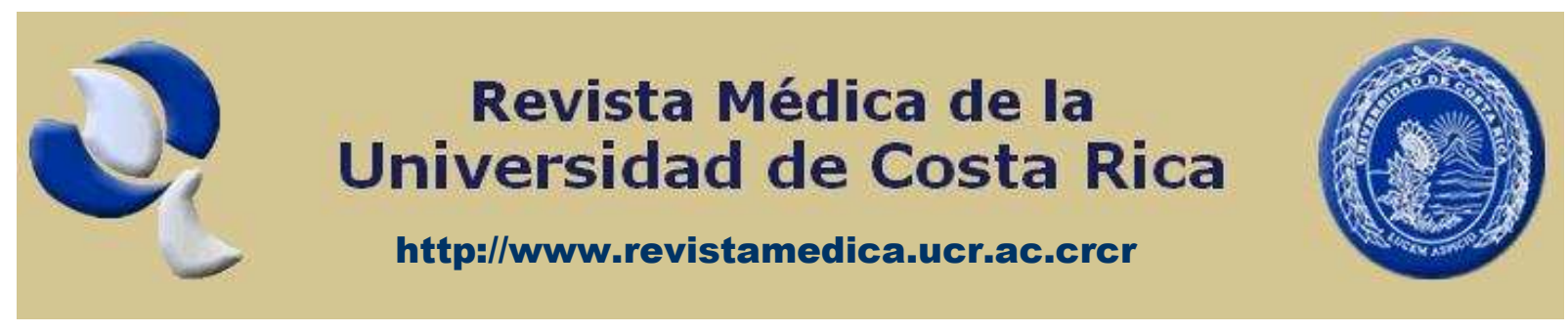

\title{
EVALUACIÓN DE LA EVIDENCIA EXISTENTE SOBRE LA EFECTIVIDAD DE LA OLANZAPINA PARA EL TRATAMIENTO DEL DELIRIUM; ANÁLISIS SISTEMÁtICO DE MEDICINA BASADA EN LA EVIDENCIA (PARTE II)
}

\section{Millán González Ricardo}

Médico psiquiatra, especialista en psiquiatría de enlace, profesor de psiquiatría de la Universidad de Costa Rica. Hospital Nacional de Geriatría y Gerontología y Centro Serenidad. San José, Costa Rica.

\section{Correspondencia: ricardo.millangonzalez@ucr.ac.cr}

\section{Resumen:}

El delirium es una alteración aguda de la consciencia y la cognición que históricamente ha sido tratada con haloperidol; debido a su perfil de efectos secundarios, recientemente se ha incrementado el uso de antipsicóticos atípicos. Objetivo: Valorar sistemáticamente la literatura sobre el empleo de la olanzapina en el tratamiento del delirium aplicando el análisis de la Medicina Basada en la Evidencia. Diseño: Se procede a identificar un problema, definir una pregunta estructurada, realizar la búsqueda de los estudios existentes y analizar objetivamente su validez y resultados. Resultados: Siguiendo una búsqueda estructurada en Medline, se detectaron cinco investigaciones que abordan el tema. Todas ellas presentan importantes carencias metodológicas y en algunos casos existen cuestionamientos éticos. Conclusiones: La 
olanzapina cuenta con un nivel 3 de evidencia clínica para su empleo como tratamiento para el delirium; se requiere de una mejoría en la estructura metodológica de estos estudios y la aplicación estricta de los principios éticos de investigación; la DRS es una escala muy difundida para cuantificar la severidad del delirium; se requiere de una lectura crítica para valorar la evidencia científica.

Palabras clave: delirium, olanzapina, medicina basada en la evidencia, antipsicóticos atípicos.

\section{Abstract:}

Delirium is an acute decline of consciousness and cognition that historically has been treated with haloperidol. Because of its side effect profile, there has been a recent increase in the use of atypical antipsychotics for this indication. Objective: To systematically evaluate the literature about the use of olanzapine in the treatment of delirium using the analysis of Evidence-Based Medicine. Design: The first step was the identification of a problem, followed by the definition of a structured question, the sistematic search of the existing studies and finally the objective analysis of the validity of the results. Results: Following a search in Medline, five studies that address the issue were identified. All of them have major methodological shortcomings and in some cases there are important ethical concerns. Conclusions: First, olanzapine has a level 3 of clinical evidence for its use as a treatment for delirium. Second, a methodological improvement in the structure of these studies is urgently required, acompanied by the strict application of the ethical principles of research. Third, the DRS is a widely used scale to quantify the severity of delirium. Forth, a critical review of the literature is essencial to assess the scientific evidence.

Key words: delirium, olanzapine, evidence-based medicine, atypical antipsychotics. 


\section{Introducción}

El delirium es una alteración de la consciencia, de carácter agudo o subagudo y patrón fluctuante, que además se acompaña de un deterioro cognitivo global, desorientación, trastornos en la percepción, déficits atencionales, aumento o disminución de la actividad psicomotriz y variaciones en el ciclo sueño-vigilia [1-3]; su identificación y tratamiento es considerada por algunos como una emergencia neurológica [4].

Hoy en día se sabe que a pesar de tratarse de un estado generalmente transitorio, sus secuelas pueden ser permanentes. Recientemente, se ha establecido una fuerte asociación entre el hecho de haber sido hospitalizado y el deterioro cognitivo posterior, siendo esta relación más fuerte cuando el ingreso se dio en una unidad de cuidados intensivos [5]. Más aún, el desarrollo de delirium en adultos mayores ha sido vinculado con un aumento posterior en la mortalidad, riesgo de institucionalización y demencia [6]

Por lo anterior y considerando su elevada prevalencia en poblaciones de pacientes hospitalizados [7], el médico tratante se ve con la responsabilidad de brindarle al paciente el mejor tratamiento existente si pretende reducir la morbimortalidad asociada con el cuadro; más aún si se considera que el tratamiento adecuado es capaz de aumentar su resolución, así como de disminuir los días de estancia hospitalaria, el número de días con delirium, y hasta los costos de la hospitalización [8].

A pesar del aumento del uso de antipsicóticos atípicos (AA) para el tratamiento del delirium [9], existen una serie de reportes del potencial de la olanzapina para inducir este cuadro [10-22]. Por esta razón, y considerando su particular perfil farmacológico, es que se desea estudiar la evidencia existente que justifique su uso en esta entidad, siguiendo el formato de Medicina Basada en la Evidencia (MBE) propuesto por Guyatt et al. [23], tal y como se aplicó previamente con la quetiapina [24].

En la presente revisión se procedió a: identificar un problema (dudas respecto a la efectividad de la olanzapina para tratar el delirium), definir una pregunta estructurada (¿Cuál es la evidencia existente de la efectividad de la olanzapina como tratamiento del delirium?), realizar una búsqueda de los estudios existentes, analizar objetiva y extensamente su validez y presentar los resultados. Se pretende así mismo promover y extender la lectura crítica como parte fundamental para la extracción de conclusiones científicas.

A continuación se detallan los pasos mencionados:

\section{Identificación de un problema: surge una duda sobre la evidencia de la efectividad de la olanzapina para tratar el delirium.}

Tal y como se comentó en la primera parte de este análisis [24], el mecanismo de acción mejor conocido de los antipsicóticos para el tratamiento del delirium se sustenta en el bloqueo de los receptores de la dopamina (D), en particular el $D_{2}$. Este neurotransmisor, gracias a una serie de metabolitos tóxicos, puede generar lesión neuronal, sobre todo en presencia de glutamato. Se sabe asimismo que la reducción farmacológica de los niveles de $D$ resulta protectora contra la hipoxia y la 
muerte neuronal, lo que restablece al menos en parte algunas de las funciones ejecutivas [25].

En las gráficas 1, 2 y 3 se muestra una adaptación de las constantes de disociación de los receptores sobre los que actúa un determinado antipsicótico, en función de su porcentaje de saturación y de la concentración plasmática (nM), según la información de su comportamiento in vitro descrito previamente por Horacek y colaboradores [26]. Como es bien conocido, los AA no ejercen una acción uniforme sobre todos los receptores; por el contrario, cada uno de ellos presenta una afinidad particular, la cual determina su perfil farmacológico.

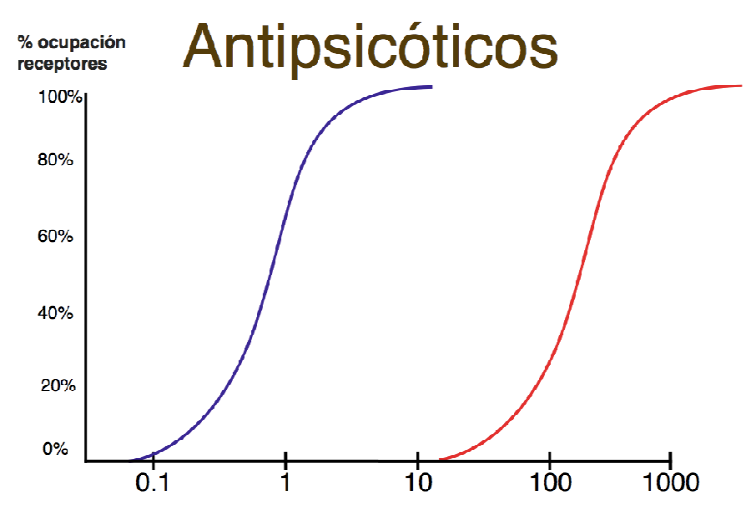

Millán 2010

Concentración (nM)

Gráfica 1: Curva genérica del porcentaje de ocupación de los receptores $A$ y $B$ en función de la concentración plasmática del antipsicótico. Toda saturación de un receptor $A$ y $B$ de un antipsicótico $X$ sigue un comportamiento en $S$, determinado por el perfil de afinidades característico de cada antipsicótico atípico. Adaptación del comportamiento in vitro descrito por Horack y colaboradores [26].

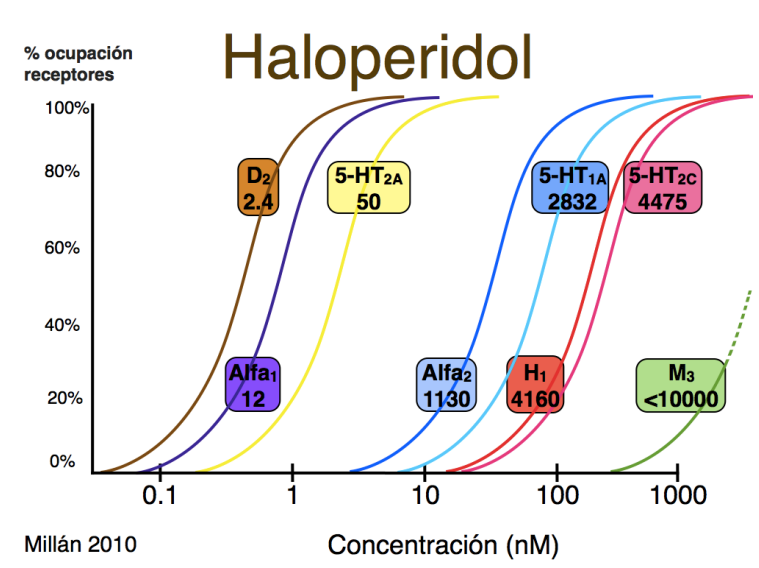

Gráfica 2: Perfil de afinidad del haloperidol por los distintos receptores sobre los que ejerce su acción. En el cuadro se anota la respectiva constante de disociación de cada uno de los receptores. Adaptación del comportamiento in vitro descrito por Horack y colaboradores [26].

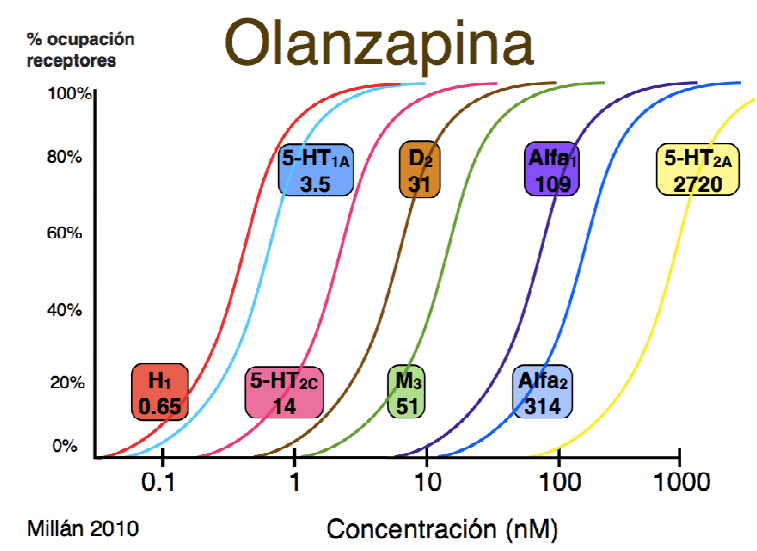

Gráfica 3: Perfil de afinidad de la olanzapina por los distintos receptores sobre los que ejerce su acción. En el cuadro se anota la respectiva constante de disociación de cada uno de los receptores. Adaptación del comportamiento in vitro descrito por Horack y colaboradores [26].

De esta manera, conforme se aumenta la concentración plasmática, en gran medida determinada por la dosis administrada, se incrementa así mismo la saturación de esos receptores, siguiendo un comportamiento en $\mathrm{S}$ (ver gráfica 1). El haloperidol ha sido tradicionalmente el fármaco de elección para el tratamiento del 
delirium debido a su alta afinidad por el receptor de $D_{2}$, lo que le permite su bloqueo incluso a dosis bajas. Es solo a dosis mayores que se obtiene un efecto sobre los receptores $\alpha_{1}, \alpha_{2}, \quad$ histaminérgicos $y$ finalmente, a dosis muy elevadas, sobre los muscarínicos (ver gráfica 2).

En el caso de la olanzapina (ver gráfica 3), su actividad sobre receptores seguirán el siguiente orden de acción conforme se asciende en la concentración plasmática: $\mathrm{H}_{1}, 5 \mathrm{HT}_{2 \mathrm{a}}, 5 \mathrm{HT}_{2 \mathrm{c}}, \mathrm{D}_{2}, \mathrm{M}_{3}, \alpha_{1}, \alpha_{2} \mathrm{~V}$ $5 \mathrm{HT}_{1 \mathrm{a}}$. Dicho patrón explica el efecto de sedación temprana en el tratamiento y el aumento del apetito, mientras que efectos antidopaminérgicos y antimuscarínicos serían observados con dosis intermedias y los efectos cardiovasculares serían observados por lo general a dosis plenas del fármaco.

En la primera parte de este análisis [24], se observó que el perfil farmacológico de la quetiapina no explica su eficacia en el tratamiento del delirium, lo que podría representar un sesgo en la interpretación de la información o el eventual descubrimiento de nuevas vías para el tratamiento para esta entidad. Considerando que el bloqueo de $D_{2}$ producido por la olanzapina ocurre en un punto intermedio de su espectro de acción, su relativa fácil disociación de este receptor [27] y su actividad anticolinérgica (ver gráfica 3), que teóricamente aumentaría la severidad del cuadro y que podría explicar los reportes de delirium inducido por este medicamento [10-22], se desea realizar un análisis sistemático de la información existente sobre su efectividad como tratamiento para el delirium.

2. ¿Cuál es la evidencia existente de la efectividad de la olanzapina como tratamiento del delirium (hiperactivo, mixto o hipoactivo) en pacientes hospitalizados según mediciones con escalas estandarizadas?

Se procedió a formular una pregunta de primer orden, en la cual se definen las características de los estudios que serán analizados. De esta manera, se abordan los siguientes aspectos:

Población: pacientes hospitalizados en centros generales, y por lo tanto con alguna patología médico-quirúrgica de fondo.

Intervención: tratamiento del delirium (hiperactivo, mixto o hipoactivo) con olanzapina sin especificación de dosis.

Desenlace: mejoría clínica cuantificada con escalas estandarizadas para la evolución del delirium.

Se deben buscar estudios randomizados y controlados (ERC) para asegurar la consistencia de la evidencia, debido a que la pregunta es de terapia o tratamiento [23].

\section{Búsqueda de la evidencia}

Se realizó una búsqueda avanzada en Medline, utilizando el siguiente formato: ("delirium"[Title/Abstract]) AND ("olanzapine"[Title/Abstract]), sin especificaciones adicionales, la cual detectó 64 artículos sobre el tema. Cuando se utilizaron términos $\mathrm{MeSH}$ o se emplearon las especificaciones de: humanos, ambos géneros, inglés y español, y personas de 19 años o mayores, no hubo artículos adicionales o no se detectaron resultados.

Los criterios de inclusión fueron: estudios en adultos hospitalizados por una condición médico-quirúrgica, de ambos 
géneros, en los que se aplicaron escalas estandarizadas para evaluar la respuesta de olanzapina en el tratamiento del delirium en cualquiera de sus variantes motoras. Los criterios de exclusión fueron estudios en idiomas distintos al español/inglés o en los que no se utilizó una escala estandarizada para cuantificar la severidad del cuadro.

Basándose en la revisión de los resúmenes de cada una de las publicaciones, se procedió a agruparlos de la siguiente manera:

- Seis escritos en idiomas distintos al español e inglés (dos en francés, uno en turco, uno en polaco, uno en italiano y uno en alemán), los cuales fueron excluidos del análisis final [28-33].

- Ocho no se enfocaban ni en el delirium ni en la olanzapina [34-41].

- Diecisiete abordaban algún aspecto de la olanzapina o antipsicóticos pero sin tener relación con el delirium [42-58].

- Nueve hacían referencia general al tratamiento del delirium con AA $[9,59-$ 66].

- En dos casos no fue posible tener acceso al documento completo, aunque no se trataba de estudios sobre la efectividad de la olanzapina en el delirium [67-68].

- Dos trataban sobre temas de delirium no relacionados con el tratamiento con olanzapina [69-70].

- Tres eran reportes de casos de tratamiento de delirium con olanzapina [71-73].

- Uno se trataba de una carta al editor comentando un artículo previamente publicado [74].

- Trece artículos describen casos de delirium inducido por el uso de la olanzapina, tres de los cuales se abordan casos de intoxicación [10-12], nueve por uso en dosis terapéuticas [13-21] y uno en el contexto de un síndrome neuroléptico maligno [22].

Cinco eran estudios específicos que abordaban la efectividad de la olanzapina como tratamiento del delirium [75-79].

4. Análisis de la validez de la evidencia

Se procedió a evaluar cada uno de los cinco estudios clínicos que abordan la eficacia de la olanzapina como tratamiento del delirium. En la tabla 1 se resumen los datos más relevantes. A continuación se expone cada uno de ellos, siguiendo su orden de antigüedad.

Sipahimalani $A$ et al. [75] realizaron un estudio prospectivo, abierto, sin randomización, en el que se comparaba la respuesta al tratamiento del delirium con dosis flexibles de olanzapina y haloperidol en dos grupos de 11 pacientes cada uno, internados en un hospital general. Se aplicó la Delirium Rating Scale (DRS) a través de valoraciones retrospectivas mediante revisión de expedientes y prospectivas durante la evolución del estudio. No se menciona la aprobación por un comité de ética ni la firma de un consentimiento informado por parte del apoderado legal. Se incluyeron pacientes con patología psiquiátrica mayor (esquizofrenia, trastorno esquizoafectivo, trastorno bipolar, depresión mayor, dependencia al alcohol y trastorno disocial de la personalidad) y tres que estaban recibiendo (y que lo continuaron haciendo) un antipsicótico típico (haloperidol en dos casos y flufenazina en otro). Se reportó una desviación estándar (DE) del DRS para la fase pretratamiento de $17.9 \pm 4.4$ para el grupo de olanzapina y de $20.1 \pm 5.2$ para el de haloperidol; así mismo, la DE 
postratamiento fue de $10.3 \pm 4.8$ para el primero de estos fármacos y $11.1 \pm 7.1$ para el segundo de ellos. Se aclara que no hubo diferencias significativas entre ambos conjuntos.

Se trata de un estudio con los siguientes inconvenientes metodológicos: un pequeño grupo de pacientes en ambos grupos (once en cada uno); el primer puntaje de la DRS se otorga de forma retrospectiva mediante revisión de expedientes; no existió randomización, por lo que la elección del antipsicótico corrió por criterio del médico tratante; no existió aprobación por un comité de ética ni se firmó un consentimiento informado para la participación; es abierto; se emplean dosis de haloperidol superiores a $3 \mathrm{mg} /$ día en promedio; no se clasifica ni cuantifica la severidad de la enfermedad médico-quirúrgica de fondo; se incluyen pacientes con patología psiquiátrica mayor y en tres usuarios se mantuvo un antipsicótico típico cuando se estaba administrando olanzapina de forma concomitante.

Kim KS et al. [76] realizaron un estudio prospectivo, abierto, no comparado, de olanzapina a dosis flexibles en 20 pacientes provenientes de una unidad de trasplante de médula ósea, de neurología y de neurocirugía, a los cuales se les había solicitado una interconsulta psiquiátrica. Para realizar el diagnóstico, se aplicaron los criterios del DSM-IV y un psiquiatra ciego al medicamento utilizado cuantificó la severidad del cuadro empleando la DRS. No se uso una escala para medir eventos adversos. Se describe una diferencia significativa $(p<0.01)$ entre la DRS inicial $(20.0 \pm 3.6)$ y la final $(9.3 \pm 4.6)$. Un total de 14 pacientes (70\%) tuvieron una mejoría superior al 50\% en la medición del DRS, 11 de los cuales pertenecían al grupo de trasplante de médula ósea. No se menciona la aprobación del estudio por parte de un comité de ética.

A pesar de que Kim KS et al. utilizan algunas consideraciones metodológicas que no se tuvieron en cuenta en el estudio anterior (consentimiento informado firmado por un familiar, exclusión de pacientes con patología psiquiátrica o que previamente o en la actualidad estuvieran recibido antipsicóticos), se trata de una investigación con las siguientes limitaciones: emplea un número muy pequeño de pacientes; es abierto y sin grupo de comparación (ya sea placebo o un estándar de oro como el haloperidol); incluye solo usuarios a quienes se les solicitó una interconsulta psiquiátrica por su "comportamiento propio del delirium"; a pesar de que los autores concluyen que el fármaco en cuestión "podría ser una opción segura para el tratamiento del delirium", no emplearon una escala estandarizada para medir efectos secundarios.

Por su parte, Breitbart W et al. [77], llevaron a cabo un estudio prospectivo, abierto, no comparativo, a dosis flexibles de olanzapina para el manejo del delirium en una población de 79 pacientes con cáncer, a quienes se les había solicitado una interconsulta psiquiátrica. El diagnostico se basó en el DSM-IV y se cuantificó utilizando la Memorial Assessment Delirium Scale (MDAS), que se fundamenta en los criterios anteriores. Asimismo, se empleó la Karnofsky Performance Status scale (KPS) para determinar el nivel de independencia funcional de los pacientes. Participaron individuos con diversos tipos de cáncer (principalmente de pulmón, gastrointestinal, 
linfoma y de mama), en diferentes estadios y se cuantificaron diferentes variables sociodemográficas y médicas, tales como edad, sexo, historia de demencia, presencia de metástasis cerebrales y la etiología del delirium. Dos entrevistadores realizaron mediciones el día que se contestó la interconsulta, al día 3 ó 4 y finalmente al día 7. Se adquirió un consentimiento verbal de participación por parte del paciente y no se presentó la investigación en un comité de ética aduciendo que los procedimientos requerían "una pequeña desviación de la práctica clínica estándar". En un inicio se solicitó intervención en un total de 154 pacientes, de los cuales 38 fueron excluidos por estar "demasiado agitados o paranoides para usar medicación oral" y otros 20 por estar ya recibiendo medicación antipsicótica. La población participante resultó ser altamente dependiente en sus habilidades de vida diaria. El análisis final detectó un descenso de la MDAS entre la primera y la segunda medición, entre la primera y la tercera y entre la segunda y la tercera, todas ellas con una diferencia estadísticamente significativa.

Se trata de una investigación en un grupo relativamente más homogéneo de pacientes (todos ellos con cáncer aunque de diferente origen y estadio), en la cual se tomaron diversas medidas sociodemográficas y clínicas, donde las evaluaciones fueron realizadas de una forma programada (al inicio, tercer o cuarto día y al sétimo día) por dos entrevistadores con una buena fiabilidad inter-evaluador y que incluyó la muestra más grande de pacientes hasta la fecha. Sin embargo, se distinguen los siguientes inconvenientes metodológicos: Se excluyó un total de 72 pacientes por diversos motivos (entre los cuales se incluye la severidad y grado de psicosis del cuadro); la medición de los efectos secundarios queda a criterio clínico de los exploradores; existe una limitación temporal del estudio (solo por 7 días); no se describe si el grupo de mayor mejoría obtuvo de forma paralela cambios de medicamentos, correcciones metabólicas y otro tipo de medidas clínicas; tampoco se cuenta con un grupo control $o$ de comparación y los exploradores no están ciegos al uso del fármaco; el consentimiento verbal se obtuvo del mismo paciente; no se puede alegar que el protocolo de este estudio no se presentó a un comité de ética por tratarse de una práctica similar a la "práctica estándar" en primer lugar porque se realizó una intervención (y de índole farmacológica) y en segundo orden porque la evidencia científica de dicha decisión clínica era muy escasa para la fecha en que se realizó la investigación.

En el estudio llevado a cabo por Skrobik et al. [78], se llevó a cabo una evaluación prospectiva, randomizada, controlada, que comparaba dosis flexibles de haloperidol y olanzapina para el tratamiento de delirium en una población 73 pacientes predominantemente quirúrgicos ingresados en una unidad de cuidados intensivos ( $\mathrm{UCl}$ ) a quienes se les había realizado una interconsulta psiquiátrica. Después de un tamizaje inicial por parte de enfermería aplicando la ICU Delirium Screening Checklist (ICU DSC), el diagnóstico lo establecía un psiquiatra aplicando los criterios del DSM-IV. Asimismo, se cuantificó la evolución clínica de los usuarios mediante el Delirium Index (DI) por considerarse más apropiada para su aplicación en ese tipo de servicios y se valoraron los efectos secundarios 
extrapiramidales empleando una de las siguientes escalas: Ross Chouinard (RC) o Simpson-Angus scale (SA). Se controlaron variables como signos vitales, función hepática, dosis diaria de antipsicóticos, dosis de "rescate" de haloperidol o benzodiacepinas en casos de agitación, y la gravedad del cuadro médico mediante la Acute Physiology and Chronic Health Evaluation (APACHE II). El estudio fue aprobado por el comité de ética institucional y se obtuvo un consentimiento informado verbal por parte de los participantes. Fue patrocinado por un fondo de Zyprexa de EliLilly Norteamérica.

Se reportó una reducción comparable entre ambos grupos de pacientes a lo largo del tiempo ( $p=0.02$ en favor del haloperidol), un uso similar de haloperidol, benzodiacepinas, opioides y sedantes no benzodiacepínicos de "rescate" y un comportamiento similar en los signos vitales y función hepática. Se empleó una dosis promedio de haloperidol de $6.5 \mathrm{mg} /$ día (rango de 1-28) y de olanzapina de 4.54 $\mathrm{mg} /$ día (rango 2.5-13.5). Se presentaron así mismo 6 pacientes en el grupo de haloperidol con efectos extrapiramidales (EEP) ( 1 evaluado con el RC y 5 con la SA), mientras que no hubo eventos adversos en el grupo de olanzapina. Por todo lo anterior los autores de la publicación concluyen que "la olanzapina constituye una alternativa segura para el haloperidol en el tratamiento de los pacientes con delirium en una $\mathrm{UCl}^{\prime \prime} \mathrm{y}$ mencionan que este es el primer estudio prospectivo y randomizado de antipsicóticos en el tratamiento del delirium en ese medio.

A pesar de lo anterior, esta investigación presenta diversas falencias que cuestionan su validez y que merecen ser mencionadas: Se incluyó una muestra con un amplio grupo etario (18-75 años); la dosis de inicio del haloperidol (7.5-15 mg/día) es muy superior a los $3 \mathrm{mg} /$ día y no necesariamente comparable con la de la olanzapina respecto al bloqueo de $D_{2}$ (ver gráficas 2, 3 y 4), lo cual resulta muy relevante por ejemplo en el paciente al que se le administró hasta 28 $\mathrm{mg} /$ día de haloperidol; se emplean benzodiacepinas como tratamiento de rescate, a pesar de su asociación con la generación de cuadros de delirium [1-2]; la randomización empleada consistió en asignar el fármaco según orden de días pares (olanzapina) o impares (haloperidol), lo que favoreció el desbalance de pacientes en favor del segundo grupo (28 contra 45, respectivamente) y podría haber generado un sesgo de apreciación por lo fácil que resulta adivinar cuál es el fármaco que está recibiendo cada usuario, más aún si se considera que los médicos y enfermeras tratantes de planta de $\mathrm{UCl}$ no estaban ciegos ante el uso del antipsicótico; el uso de "rescate" con haloperidol en ambos grupos puede ser un factor de confusión y además un elemento aditivo de riesgo de extrapiramidalismo; hubo una importante pérdida de pacientes que inicialmente podrían haber participado (214 elegibles, tan solo 80 dieron consentimiento y 73 que finalizaron el estudio); a pesar de que los grupos de edad no tienen diferencias significativas entre ellos, el rango del haloperidol (63.26 \pm 11.66$)$ es mucho más amplio que el de olanzapina (67.50 \pm 6.04$)$, lo que le confiere mayor riesgo a los pacientes más longevos del primer grupo a presentar EEP; a pesar de que no se describen diferencias significativas entre el tipo de admisión (por analizarse en conjunto), es 
claro que existe una mayoría definitiva de ingresos por cirugías de emergencia al grupo de pacientes con haloperidol (17 vs 4), lo que de nuevo, le confiere mayor riesgo a estos usuarios de presentar eventos adversos; de hecho, no se caracteriza el grupo de pacientes con EEP (edad, dosis de haloperidol/día, tipo de ingreso, edad, dosis de "rescate" con haloperidol, etc.); por si fuera poco, las escalas que cuantificaron los EEP han sido validadas para su empleo en pacientes esquizofrénicos que reciben antipsicóticos y que no cuentan con restricciones físicas ni del estado de conciencia para realizar la evaluación: por ejemplo la SA considera aspectos como la marcha, la caída del brazo y de la cabeza, la movilidad de los hombros, la rigidez de los codos, la "pendulosidad" de las piernas, y todos estos pasos de un examen físico son difícilmente realizables y con toda seguridad poco apropiados para su aplicación en una UCI. Finalmente, a pesar de ser el primer estudio que fue aprobado por un comité de ética, se admite como válido el consentimiento verbal de un usuario en delirium.

Finalmente, Kim SW et al. [79] realizó recientemente un estudio de 7 días, randomizado y comparativo a nivel clínico, entre risperidona y olanzapina, en el cual participaron 32 pacientes con una media de edad de 70 años y un predominio de diagnóstico de cáncer. Diecisiete sujetos fueron tratados con el primer fármaco y 15 con el segundo. De ellos, 12 y 8 individuos, respectivamente, terminaron el estudio debido a pérdida de seguimiento por egreso hospitalario y rechazo del consentimiento informado. Del primer grupo, 7 pacientes recibieron un total de 16 dosis de rescate de benzodiacepinas vía intramuscular, mientras que del segundo lo hicieron un total de 4 individuos en 7 oportunidades. No se especifica el método de randomización. El seguimiento lo realizó un psiquiatra ciego al tratamiento, aplicando la DRS en su versión revisado en 1998 (DRS-R-98), mientras que un segundo psiquiatra manejó la medicación a criterio clínico, sin un rango específico de dosis. Se excluyeron usuarios con demencia, trastornos hepáticos, discrasias sanguíneas o uso previo de antipsicóticos. El estudio fue aprobado por el comité de ética institucional y se obtuvo un consentimiento informado por parte de los pacientes y de los familiares.

Las características demográficas como edad, género, diagnóstico de base y puntaje basal del DRS-R-98 no mostraron diferencias significativas. Se reportó una mejoría significativa en ambos grupos al comparar los puntajes iniciales con los finales, sin que se lograra detectar diferencias significativas entre ambos grupos. El porcentaje de respuesta en el grupo de risperidona fue de $64.7 \%$ mientras que en el de olanzapina fue de $73.3 \% \quad(p=0.712)$. El tiempo medio de respuesta fue de 5 días en el primer grupo y de 3 en el segundo, nuevamente, sin diferencias significativas entre ellos. Los autores reportan ausencia de diferencias por género, pero sí una menor respuesta a risperidona en mayores de 70 años, en comparación con olanzapina en ese mismo subgrupo, con una diferencia significativa $(p=0.009)$. Ambos fármacos fueron bien tolerados. Se reportó tremor y bradiquinesia en dos pacientes del grupo de risperidona y en uno del de olanzapina, en el cual también se presentó acatisia en dos individuos adicionales; lo anterior se obtuvo 
mediante criterio clínico y no a través de la aplicación de una escala estandarizada.

Se trata de una investigación en pacientes en su mayoría adultos mayores y con diagnóstico de cáncer (no se especifica el tipo), con similares características sociodemográficas en ambos grupos. Se distinguen en todo caso, las siguientes carencias metodológicas: una muestra sumamente pequeña de pacientes de inicio, y una pérdida del seguimiento del $37.5 \%$ de quienes iniciaron ( $\tan$ solo $12(70.6 \%)$ en el grupo de risperidona y 8 (53.3\%) en el de olanzapina finalizaron); no se controló el uso de dosis de rescate de benzodiacepinas y haloperidol; no se estandarizó el rango de dosis empleado de ambos fármacos estudiados; no se clasificaron los diversos tipos de patologías ni la existencia de metástasis en sistema nervioso central; no se especificó los criterios para la randomización de los grupos; se obtuvo, al menos en una parte de la muestra, el consentimiento informado por parte de los pacientes; no se utilizó una escala estandarizada para cuantificar los efectos secundarios; y finalmente, llama poderosamente la atención que la tabla demográfica fue realizada tomando en cuenta el número de pacientes que inició el estudio y no los que lo terminaron, lo que deja la gran duda de si los análisis fueron realizados con este primero grupo, más aún considerando que se utilizó el last observation carried forward (LOCF) como parte de la revisión estadística.

\section{RESULTADOS}

Al analizar la información disponible, destacan varios factores en común de los estudios existentes:
Existe un solo ERC [78] que a pesar de un mejor planteamiento metodológico inicial que el resto de investigaciones, presenta una importante serie de sesgos que cuestionan fuertemente su validez. Las investigaciones que no son ERC presentan el inconveniente de que al conocerse el diagnóstico de delirium, sería esperable que el equipo tratante mejore una serie de parámetros que tendrán una repercusión en la resolución del cuadro y deja por fuera el carácter autolimitado de algunos de ellos; lo anterior también es cierto para la interpretación y cuantificación de eventos adversos. Para descartar la interferencia de este elemento se debe usar una comparación contra placebo y de este modo evitar un Error Tipo I donde la mejoría del cuadro esté generada por circunstancias externas y se asuma que es por el tratamiento evaluado.

Existe un solo estudio [75] que compara la olanzapina con el estándar de oro (haloperidol), pero es carente de randomización, es abierto, y cuenta con un bajo número de participantes, lo que entre otras consideraciones, pone en entredicho su validez.

Salvo en el cuarto estudio [78], no se controlan adecuadamente las variables médicas ni se contempla el grado de severidad del paciente. A pesar de que en los estudios que incluyeron pacientes con cáncer se menciona que algunos de ellos se encontraban en estado terminal, no se especifica a cuál randomización pertenecían estos individuos; puesto que es conocido que en pacientes con cáncer durante la recta final de la vida la prevalencia del delirium es cercana al 
$85 \%[1,7]$, es imperativo contemplar estos elementos a la hora de evaluar el cuadro demográfico de un estudio. Solo en uno de ellos [77] se describió la presencia de metástasis cerebrales.

En un caso [75] no se excluyeron pacientes con patología psiquiátrica mayor, lo que puede generar confusión a la hora de interpretar los síntomas del delirium. Esa misma investigación tuvo asimismo una parte retrospectiva y otra prospectiva, lo que dificulta aún más su interpretación.

No existe uniformidad respecto al modo de manejar la agitación en el caso de que la medicación estudiada no logre controlarla. De esta manera, algunos aceptan el uso de benzodiacepinas de rescate (lo que se sabe aumenta posteriormente el estado de confusión) [78-79], mientras que otros excluyen a esos pacientes (lo que podría generar un sesgo de selección y dejar pacientes con cuadros más leves dentro del estudio) [77]. Otros no consideran esta variable [75-76].

Cuando la agitación se maneja con haloperidol de rescate, se induce un factor de confusión muy alto, sobre todo si se cuantifican los EEP, tal y como ocurrió en la investigación de Skrobik et al. [78] y de Kim SW [79]. Lo anterior es aún más cierto si los pacientes ya estaban recibiendo un antipsicótico por una patología psiquiátrica mayor, tal y como ocurrió en el estudio de Sipahimalani A et al. [75].

La conclusión de que el haloperidol tiene mayor potencial de generar cuadros de extrapiramidalismo y que por lo tanto la olanzapina es más segura, solo podría realizarse si existe una equivalencia en el grado de saturación del receptor $D_{2}$ en ambos grupos, incluso después de ajustar las dosis del antipsicótico de rescate. Dosis superiores de $3 \mathrm{mg} / \mathrm{día}$ de haloperidol en el tratamiento del delirium ponen al paciente en riesgo de reacciones adversas extrapiramidales [9], por una saturación de los receptores de $\mathrm{D}_{2}$ superior al $80 \%$ (ver gráfica 4 ). Por lo tanto, las dosis empleadas en el estudio de Skrobik et al. [78], con un promedio de haloperidol de $6.5 \mathrm{mg} /$ día (rango de 128) y de olanzapina de $4.54 \mathrm{mg} /$ día (rango 2.5-13.5), no son equivalentes en su actividad sobre $D_{2}$ y no es posible apoyar la tesis del menor perfil de efectos adversos del segundo de estos fármacos. Ocurre algo similar en el estudio de Sipahimalani A et al. [75].

Las muestras presentadas están en el rango de 22-96 pacientes que iniciaron estudios (total de 239) y entre 11-79 que los terminaron (total de 203), divididos en 5 investigaciones. Lo anterior sin contar que en el tercer estudio [77] inicialmente se respondió la interconsulta de 154 pacientes, de los cuales finalizaron el estudio tan solo 79; asimismo la exclusión inicial de pacientes con cuadros de mayor severidad podría condicionar las conclusiones de la investigación a un grupo de pacientes con cuadros de leve o moderada intensidad únicamente.

La pérdida del seguimiento es un factor de confusión porque se desconoce el tipo de evolución que esos individuos podrían haber tenido, ya sea positiva o negativa [80]. Las poblaciones pequeñas participantes en los estudios, sobre todo 
cuando son menores de 100 pacientes [81], complican los cálculos estadísticos y la generalización de los hallazgos.

Todos los estudios incluyeron pacientes que fueron referidos para interconsultas psiquiátricas. Es bien conocida la tendencia a que estados de delirium hipoactivo pasen desapercibidos [7] y por lo tanto que generen menos referencias a médicos especializados en el tema, lo que podría generar una menor representación de esta variante del cuadro.

- A pesar del cuestionamiento actual de su validez [82], no se diferenciaron las variables motoras del delirium. Dadas las características sedantes de la olanzapina y lo descrito en el punto anterior, este elemento eventualmente podría ser de utilidad clínica.

Tres de los cinco estudios revisados emplearon la DRS o la DRS-R98. Si se considera que todos los estudios que revisaron la eficacia de la quetiapina [24] utilizaron solo las dos primeras escalas en cuestión, se hace evidente que son herramientas muy difundidas para evolucionar y cuantificar la severidad del delirium.

- En general, se reportaron escasos efectos adversos, sin embargo en algunos estudios no se utilizó una escala estandarizada para su cuantificación [7577, 79], mientras que en otros se emplearon herramientas no adecuadas para la población ni el medio en que se realizó la exploración [78].

El estudio de Breitbart et al. [77] no se presenta la medición de la MDAS al inicio $y$ al final del estudio, y por lo tanto no existen diferencias entre uno y otro valor.
Los autores de esa investigación se centraron más en el perfil de efectos adversos que en el objetivo primario, lo que representa un factor de confusión en la lectura de su artículo.

Dos estudios [77, 79] fueron diseñados para durar 7 días, asumiendo que para ese momento el cuadro podría estar resuelto. A pesar de que en muchos de los casos puede darse un control temprano de los síntomas, lo anterior no ocurre en todos los pacientes. Esa limitación en tiempo del estudio impide evolucionar a largo plazo a los participantes, por lo que se desconoce efectos posteriores a la primera semana después de iniciado el tratamiento.

Llama la atención que en tres estudios no hubo aprobación de un comité de ética [75-77], incluso a pesar de que en el primero de ellos participaron pacientes vulnerables por presentar una patología psiquiátrica mayor; en el tercero [77] se justifica la ausencia de dicho trámite debido a la "pequeña desviación de la práctica clínica estándar", razonamiento incongruente con la escasa información científica disponible sobre la efectividad de la olanzapina en ese entonces, lo que atenta contra los principios básicos de la investigación. En tres oportunidades [7779], a pesar de que dos de ellas se realizaron con aprobación por un comité de ética, el consentimiento informado fue brindado de forma verbal por el paciente, excepto en el último de ellos en que el documento fue consentido por el paciente o por el apoderado legal. Puesto que el estado de delirium por definición contempla una fluctuación del estado de consciencia y un compromiso de las 
funciones cognitivas [1-3], el juicio del paciente siempre estará comprometido y por lo tanto se verá incapacitado para tomar decisiones, entre las que se incluye el brindar un consentimiento informado.

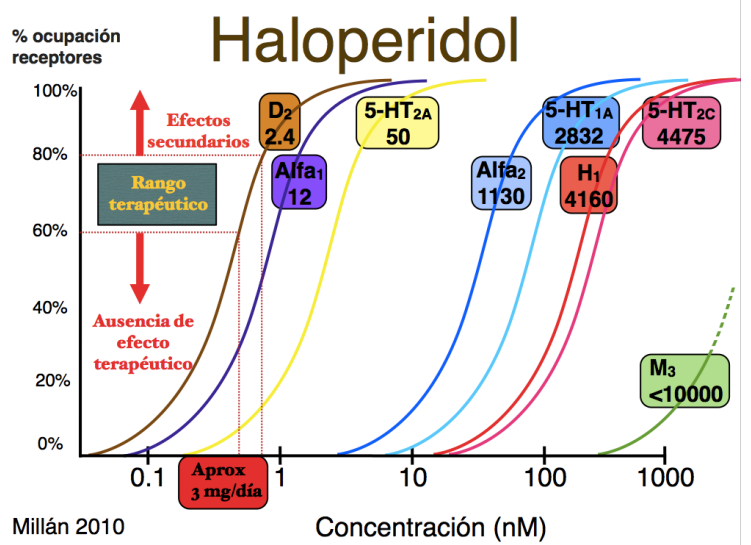

Gráfica 4: Margen de ocupación de receptores de $D_{2}$ necesarios para alcanzar un adecuado rango terapéutico y su correspondiente dosis oral aproximada por día. Adaptación del comportamiento in vitro descrito por Horack y colaboradores [26].

El nivel jerárquico de la MBE propone clasificar la evidencia en niveles, de acuerdo con su estructura metodológica. De esta manera, el nivel 1 se obtiene cuando una determinada práctica tiene un respaldo sustentado en varios ERC con una adecuada metodología que además han replicados, el nivel 2 ocurre cuando existe un solo ERC sin replicación o comparación activa pero conservando el doble ciego y una correcta aplicación metodológica estándar, el nivel 3 se da cuando existen estudios pequeños prospectivos no controlados con más de 10 sujetos y el nivel 4 es propio de reportes anecdóticos o de casos clínicos.

Aunque existe una investigación con un diseño de ERC [78], su validez es fuertemente cuestionada por el actual análisis; los restantes tampoco tienen un diseño de ERC y presentan asimismo una serie de sesgos que ya han sido mencionados. Por lo tanto, el empleo de la olanzapina para el tratamiento de delirium en este momento cuenta tan solo con un nivel 3 de evidencia clínica: 5 estudios prospectivos no controlados con más de 10 sujetos [75-79], y algunos reportes de casos [71-73].

Al igual que en el caso de la quetiapina, es posible que existan mecanismos distintos a los postulados tradicionalmente para el manejo del delirium (bloqueo de $\mathrm{D}_{2}$ ). Tal como se mencionó en la primera parte de este análisis, la quetiapina podría mejorar el patrón de sueño y de la alteración del ritmo circadiano [82] a través del bloqueo de histamina y del receptor del $5 \mathrm{HT}_{2}$ (que aumenta el sueño de ondas lentas) [83], lo que también podría ocurrir con la olanzapina dado su perfil farmacológico en bajas concentraciones plasmáticas (ver gráfica 3). Cabe recordar que el comportamiento de estos fármacos in vivo no necesariamente corresponde con el establecido a nivel in vitro, por lo que podría darse un temprano bloqueo de $D_{2}$ y esto justificaría su acción sobre el delirium. De hecho esta es la justificación para pensar que incluso a dosis terapéuticas se puede observar casos de delirium inducido por este fármaco [10-22], presumiblemente por su temprana acción anticolinérgica.

Como inconvenientes de la actual revisión se menciona que la búsqueda se limitó a una sola base de datos y que no se realizó una traducción de los artículos en idiomas distintos al español/inglés; por lo anterior, podría existir información que no ha sido contemplada en este análisis. 


\section{CONCLUSIONES}

1. En el momento actual, la olanzapina cuenta con un nivel 3 de evidencia clínica para su empleo como tratamiento para el delirium.

2. Se requieren ERC con un robusto diseño metodológico que investiguen la efectividad de la olanzapina para el tratamiento de delirium en poblaciones extensas. Entre las variables que se deben contemplar se mencionan: un adecuado estadiaje de la patología médico-quirúrgica (incluyendo la presencia de deterioro cognitivo previo) y de su severidad y complicaciones (como la presencia de metástasis en el caso de pacientes con diversos tipos de cáncer); la aplicación del estudio en una población determinada que fue tamizada por delirium (sin el sesgo de referencia mediante interconsulta); el adecuado control de variables farmacológicas y de ambiente; la correcta selección de las herramientas de medición de los efectos adversos (que deben ser aplicables en la población y en el ambiente determinado); la exclusión de factores de confusión como tratamientos de rescate o concomitante (ya sean benzodiacepinas - antipsicóticos) y la presencia de patología psiquiátrica mayor; el empleo de dosis comparables en cuanto a su potencial de saturación de los receptores de $D_{2}$ entre la olanzapina y el antipsicótico de comparación; por tratarse de pacientes con una patología médico-quirúrgica de fondo, en caso de que existan defunciones, estas deben ser reportadas, así como su causa; se debe también estandarizar las dosis de los antipsicóticos utilizados.
3. Es indispensable el adecuado empleo de los principios éticos de la investigación: en un estudio intervencionista, en el cual se administran fármacos que no tienen una robusta evidencia científica hasta la fecha (en el caso del delirium), resulta fundamental el obtener la aprobación de un comité de ética certificado. De igual manera, cuando se trata de una patología que por definición compromete el estado de consciencia y las facultades cognitivas del individuo, el consentimiento informado deberá ser obtenido por un representante legal competente mentalmente y nunca por el paciente.

4. La DRS sigue siendo una escala muy difundida para valorar la severidad y evolución de los pacientes con delirium.

5. Tal y como se comenta en la primera parte de este análisis, es necesaria una lectura crítica de los diversos estudios que se difunden en las bases de datos. Una presentación metodológica elegante no necesariamente asegura la ausencia de sesgos. 


\section{BIBLIOGRAFÍA}

1. Maldonado JR. Delirium in the Acute Care Setting: Characteristics, Diagnosis and Treatment. Crit Care Clin 2008; 24: 657722.

2. Maldonado JR. Pathoetiological Model of Delirium: a Comprehensive Understanding of the Neurobiology of Delirium and an Evidence-Based Approach to Prevention and Treatment. Crit Care Clin 2008; 24: 789856.

3. American Psychiatric Association. Diagnostic and Statistical Manual of Mental Disorders: DSM-IV-TR, Fourth Edition (Text Revision). IV ed.: American Psychiatric Press; 2000.

4. Douglas VC, Josephson SA. Delirium. Continuum Lifelong Learning Neurol 2010; 16 (2): 120-134.

5. Ehlenbach WJ, Hough CL, Crane PK, Haneuse S, Carson SS, Curtis JR, Larson EB. Association Between Acute Care and Critical Illness Hospitalization and Cognitive Function in Older Adults. JAMA 2010; 303 (8): 763-770.

6. Witlox J, Eurelings LSM, de Jonghe JFM, Kalisvaart KJ, Eikelenboom P, van Gool WA. Delirium in Elderly Patients and the Risk of Postdischarge Mortality, Institutionalization, and Dementia: A Metaanalysis. JAMA 2010; 304 (4): 443-451.

7. Liptzin B. Clinical Diagnosis and Management of Delirium. In: Stoudemire A, Fogel BS, Greenberg D, editors. Psychiatric Care of Medical Patients. Second Ed. New York, NY: OxfordUniversity Press, Inc.; 2000. p. 581-596.

8. Maldonado JR, Dhami N. Recognition and management of delirium in the medical and surgical intensive care wards. Journal of Psychosomatic Research 2003;55(2):150.

9. Lonergan E, Britton AM, Luxemberg J. Antipsychotics for Delirium (Review). The Cochrane Colaboration 2009; 1: 1-18.

10. Morgan M, Hackett LP, Isbister GK. Olanzapine overdose: a series of analytically confirmed cases. Int Clin Psychopharmacol 2007; 22 (3): 183-186.

11. Aurora M, Praharaj SK. Nonfatal suicidal olanzapine overdose: a case report. Clin Neuropharmacol 2006; 29(4): 190-191.

12. Weizberg M, Su M, Mazzola JL, Bird SB, Brush DE, Boyer EW. Altered mental status from olanzapine overdose treated with physostigmine. Clin Toxicol (Phila) 2006; 44(3):319-325.

13. Detke HC, McDonnell DP, Brunner E, Zhao F, Sorsaburu S, Stefaniak VJ, Corya SA.Postinjection delirium/sedation syndrome in patients with schizophrenia treated with olanzapine long-acting injection, I: analysis of cases. BMC Psychiatry 2010; 10(10):43.

14. McDonnell DP, Detke HC, Bergstrom RF, Kothare $P$, Johnson J, Stickelmeyer $M$, Sanchez-Felix MV, Sorsaburu S, Mitchell MI. Post-injection delirium/sedation syndrome in patients with schizophrenia treated with olanzapine long-acting injection, II: investigations of mechanism. BMC Psychiatry 2010; 10(10):45.

15. Sharma RC, Aggarwal A. Delirium associated with olanzapine therapy in an elderly male with bipolar affective disorder. Psychiatry Investig 2010; 7(2): 153-154.

16. Lim CJ, Trevino C, Tampi RR. Can olanzapine cause delirium in the elderly? Ann Pharmacother 2006; 40(1): 135-138.

17. Tuglu C, Erdogan E, Abay E. Delirium and extrapyramidal symptoms due to a lithiumolanzapine combination therapy: a case report. J Korean Med Sci 2005; 20(4): 691694.

18. Estfan B, Yavuzsen T, Davis M. Development of opioid-induced delirium while on olanzapine: a two-case report. I Pain Symptom Manage 2005; 29(4): 330-332.

19. Morita $T$, Tei $Y$, Shishido $H$, Inoue $S$. Olanzapine-induced delirium in a terminally ill cancer patient. J Pain Symptom Manage 2004; 28(2): 102-103. 
20. Samuels S, Fang M. Olanzapine may cause delirium in geriatric patients. I Clin Psychiatry 2004; 65(4): 582-583.

21. Robinson RL, Burk MS, Raman S. Fever, delirium, autonomic instability, and monocytosis associated with olanzapine. J Postgrad Med 2003; 49(1): 96.

22. Arnaout MS, Antun FP, Ashkar K. Neuroleptic malignant syndrome with olanzapine associated with severe hypernatremia. Hum Psychopharmacol 2001; 16(3): 279-281.

23. Guyatt G, Rennie D, Meade MO, Cook DJ. JAMA evidence: Users' guidelines to the medical literature. Second Ed. New York, New York: McGraw-Hill Medical; 2008.

24. Millán-González R. Evaluación de la evidencia existente sobre la efectividad de la quetiapina para el tratamiento del delirium: Análisis sistemático de Medicina Basada en la Evidencia. Revista Médica de la Universidad de Costa Rica 2010; 4 (1): 2333.

25. Brown TM. Basic Mechanisms in the Pathogenesis of Delirium. In: Stoudemire A, Fogel BS, Greenberg D, editors. Psychiatric Care of the Medical Patient. Second Ed. New York, NY: Oxford University Press, Inc.; 2000. p. 571-580.

26. Horacek J, Bubenikova-Valesova V, Kopecek M, Palenicek T, Dockery C, Mohr P, Hoschl C. Mechanism of Action of Atypical Antipsychotic Drugs and the Neurobiology of Schizophrenia. CNS Drugs 2006; 20 (5): 389-409.

27. Seeman P. Atypical Antypsychotics: Mechanism of Action. Can J Psychiatry 2002; 47: 27-38.

28. Pelland C, Trudel JF. Atypical antipsychotic efficacy and safety in managing delirium: a systematic review and critical analysis. Psychol Neuropsychiatr Vieil 2009; 7(2):109119.

29. Patry L, Guillem E, Pontonnier F, Ferreri M. Catatonia de novo, report on a case: immediate vital prognosis and psychiatric prognosis in longer term. Encephale 2003; 29(1): 72-79.

30. Onur E, Cimilli C. A new approach to the treatment of delirium: atypical antipsychotics. Turk Psikiyatri Derg 2005;16 (3): 216-224.

31. Magdalan J, Wasyko-Smolarek $M$, Antończyk A, Kochman K, Przewłocki M, Porebska B. Intoxications with atypical antipsychotic drugs--data of the Acute Intoxication Unit in Wrocław. Przegl Lek 2004; 61 (4): 392-396.

32. Riva E, Nobili A, Trecate F. "Judicious" use of neuroleptic drugs in the treatment of behavioral symptoms in the course of Alzheimer disease. Recenti Prog Med 1998; 89(11): 598-603.

33. Steil B. A case of delirium after olanzapine intoxication. Nervenarzt 2003; 74(11):10091011.

34. Gulsun M, Pinar M, Sabanci U. Psychotic disorder induced by oxybutynin: Presentation of two cases. Clin Drug Investig 2006; 26 (10): 603-606.

35. Foley KF, Kast RE. Review of evidence that posttransplantation psychiatric treatment commonly affects prolactin levels and thereby influences graft fate. Gen Hosp Psychiatry 2006; 28 (3): 230-233.

36. Lauterbach EC. The neuropsychiatry of Parkinson's disease and related disorders. Psychiatr Clin North Am 2004; 27 (4):801825.

37. Fernandez $\mathrm{HH}$, Trieschmann ME, Friedman $\mathrm{JH}$. Treatment of psychosis in Parkinson's disease: safety considerations. Drug Saf 2003; 26 (9): 643-659.

38. Kuzuhara S. Drug-induced psychotic symptoms in Parkinson's disease. Problems, management and dilemma. J Neurol 2001; 248 (Suppl 3): 28-31.

39. Wolters EC, Berendse HW. Management of psychosis in Parkinson's disease. Curr Opin Neurol 2001; 14 (4): 499-504. 
40. Melamed E, Friedberg G, Zoldan J. Psychosis: impact on the patient and family. Neurology $1999 ; 52$ [7 (Suppl 3)]: S14-16.

41. Zannino G, Gargiulo A, Lamenza F, Marotta MG, Barzotti T, Silvestri A, Ettorre E, Marigliano $V$. The management of psychogeriatric patient. Arch Gerontol Geriatr Suppl 2004; (9): 465-470.

42. Citrome L. Olanzapine pamoate: a stick in time? A review of the efficacy and safety profile of a new depot formulation of a second-generation antipsychotic. Int J Clin Pract 2009; 63 (1): 140-150.

43. Kane JM, Detke HC, Naber D, Sethuraman G, Lin DY, Bergstrom RF, McDonnell D. Olanzapine long-acting injection: a 24-week, randomized, double-blind trial of maintenance treatment in patients with schizophrenia. Am J Psychiatry 2010; 167 (2):181-189.

44. Citrome L. Patient perspectives in the development and use of long-acting antipsychotics in schizophrenia: focus on olanzapine long-acting injection. Patient Prefer Adherence 2009; 29 (3): 345-355.

45. Owen RT. Olanzapine: a review of rapid and long-acting parenteral formulations. Drugs Today (Barc) 2010; 46 (3): 173-181.

46. Fleischhacker WW. Second-generation antipsychotic long-acting injections: systematic review. Br J Psychiatry Suppl 2009; 52: S29-36.

47. Lindenmayer J. Long-acting injectable antipsychotics: focus on olanzapine pamoate. Neuropsychiatr Dis Treat 2010; 24 (6): 261-267.

48. Cañas F, Möller HJ. Long-acting atypical injectable antipsychotics in the treatment of schizophrenia: safety and tolerability review. Expert Opin Drug Saf 2010; Aug 8.

49. Perlis RH, Baker RW, Zarate CA Jr, Brown EB, Schuh LM, Jamal HH, Tohen M. Olanzapine versus risperidone in the treatment of manic or mixed States in bipolar I disorder: a randomized, double- blind trial. J Clin Psychiatry 2006; 67 (11): 1747-1753.

50. Nielsen J, Bruhn AM. Atypical neuroleptic malignant syndrome caused by olanzapine. Acta Psychiatr Scand 2005; 112 (3): 238240.

51. Duggal MK, Singh A, Arunabh, Lolis JD, Guzik HJ. Olanzapine-induced vasculitis. Am J Geriatr Pharmacother 2005; 3 (1): 21-24.

52. Alexopoulos GS, Streim J, Carpenter D, Docherty JP; Expert Consensus Panel for Using Antipsychotic Drugs in Older Patients. Using antipsychotic agents in older patients. J Clin Psychiatry 2004; 65 Suppl 2: 5-99.

53. Davis MP, Khawam E, Pozuelo L, Lagman R. Management of symptoms associated with advanced cancer: olanzapine and mirtazapine. A World Health Organization project. Expert Rev Anticancer Ther 2002; 2 (4): 365-376.

54. Khojainova N, Santiago-Palma J, Kornick C, Breitbart W, Gonzales GR. Olanzapine in the management of cancer pain. J Pain Symptom Manage 2002; 23 (4): 346-350.

55. Kennedy JS, Zagar A, Bymaster F, Nomikos G, Trzepacz PT, Gilmore JA, Rotelli MD, Breier A, Tollefson G. The central cholinergic system profile of olanzapine compared with placebo in Alzheimer's disease. Int J Geriatr Psychiatry 2001; 16 Suppl 1: S24-32.

56. Winans EA, Janicak PG. IM olanzapine in the treatment of agitation and aggression. Expert Rev Neurother 2001; 1 (1): 28-32.

57. Davis MP, Dickerson ED. Olanzapine: another psychotropic? Am J Hosp Palliat Care 2001; 18 (2): 129-132.

58. Solomons K, Geiger O. Olanzapine use in the elderly: a retrospective analysis. Can J Psychiatry. 2000 Mar;45(2):151-5.

59. Seitz DP, Gill SS, van Zyl LT. Antipsychotics in the treatment of delirium: a systematic review. J Clin Psychiatry 2007; 68 (1): 11-21.

60. Rea RS, Battistone S, Fong JJ, Devlin JW. Atypical antipsychotics versus haloperidol for treatment of delirium in acutely ill 
patients. Pharmacotherapy 2007; 27 (4): 588-594.

61. Ozbolt LB, Paniagua MA, Kaiser RM. Atypical antipsychotics for the treatment of delirious elders. J Am Med Dir Assoc 2008; 9 (1): 18-28.

62. Attard A, Ranjith G, Taylor D. Delirium and its treatment. CNS Drugs 2008; 22 (8):631644.

63. Gagnon PR. Treatment of delirium in supportive and palliative care. Curr Opin Support Palliat Care. 2008; 2 (1): 60-66.

64. Lacasse $H$, Perreault MM, Williamson DR. Systematic review of antipsychotics for the treatment of hospital-associated delirium in medically or surgically ill patients. Ann Pharmacother 2006; 40 (11): 1966-1973.

65. Boettger S, Breitbart W. Atypical antipsychotics in the management of delirium: a review of the empirical literature. Palliat Support Care 2005; 3 (3): 227-237.

66. Tune L. The role of antipsychotics in treating delirium. Curr Psychiatry Rep 2002; 4 (3):209-212.

67. Singh D, Forlano R. Olanzapine and delirium: lesson from clinical practice. Aust N Z J Psychiatry 2008; 42(5): 433-434.

68. Gupta N, Sharma P, Prabhakar S. Olanzapine for delirium in parkinsonism: therapeutic benefits in lieu of adverse consequences. Neurol India 2004; 52(2): 274-275.

69. Centeno C, Sanz A, Bruera E. Delirium in advanced cancer patients. Palliat Med 2004; 18(3): 184-194.

70. Takeuchi T, Furuta K, Hirasawa T, Masaki H, Yukizane $\mathrm{T}$, Atsuta $\mathrm{H}$, Nishikawa $\mathrm{T}$. Perospirone in the treatment of patients with delirium. Psychiatry Clin Neurosci 2007; 61(1): 67-70.

71. Halil M, Cankurtaran ES, Ozkayar N, Cankurtaran M, Ulger Z, Yavuz BB, Shorbagi A, Ariogul S. Elderly patient with delirium after myocardial infarction.J Natl Med Assoc 2006; 98(4): 648-650.
72. Passik SD, Cooper M. Complicated delirium in a cancer patient successfully treated with olanzapine. J Pain Symptom Manage 1999; 17(3): 219-223.

73. Krieger D, Hansen K, McDermott C, Matthews R, Mitchell R, Bollegala $\mathrm{N}$, Bhalerao $S$. Loxapine versus olanzapine in the treatment of delirium following traumatic brain injury. NeuroRehabilitation 2003; 18(3): 205-208.

74. Prommer E. Re: Olanzapine-induced delirium. J Pain Symptom Manage 2005; 29(2):119-120.

75. Sipahimalani A, Masand PS. Olanzapine in the treatment of delirium. Psychosomatics 1998; 39(5): 422-430.

76. Kim KS, Pae CU, Chae JH, Bahk WM, Jun T. An open pilot trial of olanzapine for delirium in the Korean population. Psychiatry Clin Neurosci 2001; 55(5): 515519.

77. Breitbart W, Tremblay A, Gibson C. An open trial of olanzapine for the treatment of delirium in hospitalized cancer patients. Psychosomatics 2002; 43(3): 175-182.

78. Skrobik YK, Bergeron N, Dumont M, Gottfried SB. Olanzapine vs haloperidol: treating delirium in a critical care setting. Intensive Care Med 2004; 30(3): 444-449.

79. Kim SW, Yoo JA, Lee SY, Kim SY, Bae KY, Yang SJ, Kim JM, Shin IS, Yoon JS. Risperidone versus olanzapine for the treatment of delirium. Hum Psychopharmacol 2010; 25(4): 298-302.

80. Gómez-Restrepo C. Curso de Medicina Basada en Evidencia. Especialidad de Psiquiatría de Enlace 2009. Pontificia Universidad Javeriana, Bogotá, Colombia.

81. Sachs G. Presentación: "Evidencia del Tratamiento del Trastorno Afectivo Bipolar". Forth Biennial Conference of The International Society of Bipolar Disorders: Grand Hyatt Hotel, 17-20 March 2010; Sao Pablo, Brazil.

82. Trzepacz P. Presentación: "Advanced Management of Delirium". American 
Psychosomatic Medicine Annual Meeting 2009. Hotel Río; Las Vegas, Nevada, Estados Unidos.

83. Sasaki Y, et al. A Prospective, Open-Label, Flexible-Dose Study of Quetiapine in the Treatment of Delirium. J Clin Psychiatry 2003; 64: 1316-132. 
84. Tabla 1. Estudios encontrados que han evaluado la efectividad de la olanzapina como tratamiento del delirium.

\begin{tabular}{|c|c|c|c|c|c|c|c|c|c|c|c|}
\hline Autor & Tipo de estudio & $\begin{array}{c}\text { Número de } \\
\text { pacientes } \\
\text { que } \\
\text { iniciaron } \\
\text { estudio }\end{array}$ & $\begin{array}{l}\text { Número de } \\
\text { pacientes } \\
\text { que } \\
\text { finalizaron } \\
\text { estudio }\end{array}$ & $\begin{array}{c}\text { Escala } \\
\text { estandarizada } \\
\text { de medición }\end{array}$ & $\begin{array}{c}\text { Criterios } \\
\text { diagnósticos } \\
\text { empleados }\end{array}$ & $\begin{array}{c}\text { Dosis } \\
\text { promedio de } \\
\text { olanzapina }\end{array}$ & $\begin{array}{l}\text { Medición inicial de } \\
\text { escala estandarizada } \\
\text { para delirium }\end{array}$ & $\begin{array}{l}\text { Medición final } \\
\text { de escala } \\
\text { estandarizada } \\
\text { para delirium }\end{array}$ & $\begin{array}{c}\text { Diferencia } \\
\text { estadísticamente } \\
\text { significativa } \\
\text { entre medición } \\
\text { inicial y final }\end{array}$ & $\begin{array}{l}\text { Aprobación } \\
\text { por comité } \\
\text { de ética }\end{array}$ & $\begin{array}{c}\text { Obtención de } \\
\text { consentimient } \\
0\end{array}$ \\
\hline $\begin{array}{l}\text { Sipahimalani A, } \\
\text { et al. } 1998\end{array}$ & $\begin{array}{c}\text { Prospectivo, } \\
\text { abierto, no } \\
\text { randomizado, } \\
\text { dosis flexible, } \\
\text { comparativo }\end{array}$ & $\begin{array}{c}11 \text { Hal vs. } \\
11 \text { Olan }\end{array}$ & $\begin{array}{l}11 \text { Hal vs. } \\
11 \text { Olan }\end{array}$ & $\begin{array}{l}\text { DRS } \\
\text { CGI }\end{array}$ & \begin{tabular}{|c|} 
No se \\
especifican
\end{tabular} & \begin{tabular}{|c|}
$8.2 \pm 3.4$ \\
mg/día \\
Olan vs. \\
$5.1 \pm 3.5$ Hal
\end{tabular} & $\begin{array}{c}19.9 \pm 4.4 \text { (Olan) vs. } \\
20.09 \pm 5.2 \text { (Hal) } \\
\text { (primer día) }\end{array}$ & $\mid \begin{array}{c}10.3 \pm 4.8 \text { (Olan) } \\
\text { vs. } 11.1 \pm 7.1 \\
\text { (Hal) (variable, } \\
\text { promedio } 7 \\
\text { días) }\end{array}$ & No se reportan & No & No \\
\hline $\begin{array}{l}\text { Kim KS, et al. } \\
2001\end{array}$ & $\begin{array}{l}\text { Prospectivo, no } \\
\text { comparado, } \\
\text { dosis flexible }\end{array}$ & 20 & 20 & DRS & DSM-IV & $5.9 \pm 1.5$ & $\begin{array}{c}20.0 \pm 3.6 \\
\text { (primer día) }\end{array}$ & $\begin{array}{c}9.3 \pm 4.6 \\
\text { (variable, } \\
\text { promedio } 6.6 \\
\text { días) }\end{array}$ & $p<0.01$ & No & $\begin{array}{l}\text { Firmado por } \\
\text { familiares }\end{array}$ \\
\hline $\begin{array}{l}\text { Breitbart W, et al. } \\
2002\end{array}$ & $\begin{array}{l}\text { Prospectivo, } \\
\text { abierto, no } \\
\text { comparado, } \\
\text { dosis flexible }\end{array}$ & \begin{tabular}{|c|}
82 \\
(previa \\
exclusión \\
de 72 \\
pacientes \\
) \\
\end{tabular} & 79 & $\begin{array}{l}\text { MDAS } \\
\text { KPS }\end{array}$ & DSM-IV & \begin{tabular}{|c|}
$3.0 \mathrm{mg} / \mathrm{dí}$ \\
(inicial); 4.6 \\
$\mathrm{mg} / \mathrm{día}$ \\
(segunda); \\
$6.3 \mathrm{mg} / \mathrm{dia} a$ \\
(tercera) \\
\end{tabular} & $\begin{array}{l}19.85 \pm 3.79 \\
\text { (primer día) }\end{array}$ & $\begin{array}{l}10.78 \pm 7.31 \\
\text { (sétimo día) }\end{array}$ & $p<0.001$ & No & $\begin{array}{l}\text { Verbal del } \\
\text { paciente }\end{array}$ \\
\hline $\begin{array}{l}\text { Skrobik, et al. } \\
2004\end{array}$ & $\begin{array}{c}\begin{array}{c}\text { Prospectivo, } \\
\text { randomizado, } \\
\text { controlado }\end{array} \\
\end{array}$ & \begin{tabular}{|c|}
80 \\
$(214$ \\
pacientes \\
elegibles $)$
\end{tabular} & 73 & $\begin{array}{l}\text { ICU-DSC } \\
\text { DI } \\
\text { RCs } \\
\text { SAs }\end{array}$ & DSM-IV & \begin{tabular}{|c|} 
Hal: 6.5 \\
$\mathrm{md} /$ día (1- \\
28); Olan: \\
$4.54 \mathrm{mg} / \mathrm{día}$ \\
$(2.5-13.5)$
\end{tabular} & No se especifica & $\begin{array}{c}\text { No se } \\
\text { especifica }\end{array}$ & \begin{tabular}{|c|} 
No se \\
especifica; no \\
hay diferencias \\
entre los \\
grupos \\
\end{tabular} & $\mathrm{Si}$ & $\begin{array}{l}\text { Verbal del } \\
\text { paciente }\end{array}$ \\
\hline $\begin{array}{l}\text { Kim SW, et al. } \\
2010\end{array}$ & $\begin{array}{l}\text { Randomizado, } \\
\text { comparativo }\end{array}$ & $\begin{array}{c}32 ; 17 \\
\text { Risp, } 15 \\
\text { Olan }\end{array}$ & $\begin{array}{c}20 ; 12 \text { Risp, } \\
8 \text { Olan }\end{array}$ & DRS-R-98 & DSM-IV & $\begin{array}{c}\text { No se } \\
\text { especifican }\end{array}$ & $\begin{array}{c}0.6 \pm 0.2 \mathrm{mg} / \mathrm{día}(0.25- \\
1 \mathrm{mg} / \mathrm{día} \mathrm{Risp}) \text { vs. } \\
1.8 \pm 0.6 \mathrm{mg} / \mathrm{día} \\
(1.25-7.5 \mathrm{mg} / \mathrm{día} \\
\text { Olan) }\end{array}$ & $\begin{array}{c}0.9 \pm 0.6 \mathrm{mg} / \mathrm{día} \\
\text { (0.25-2 mg/día } \\
\text { Risp) vs. } \\
2.4 \pm 1.7 \mathrm{mg} / \mathrm{dí} a \\
(1.25-7.5 \\
\mathrm{mg} / \mathrm{dí} \text { OOlan) }\end{array}$ & \begin{tabular}{|c|} 
Sin diferencias \\
estadísticas \\
entre grupos; \\
con diferencia \\
significativa \\
entre puntaje \\
inicial y final en \\
ambos grupos \\
$(p<0.01)$
\end{tabular} & $\mathrm{Si}$ & $\begin{array}{c}\text { Del paciente } \\
\text { y del familiar } \\
\text { (predominant } \\
\text { emente) }\end{array}$ \\
\hline
\end{tabular}

Hal: haloperidol; Olan: olanzapina; Risp: risperidona; DRS: Delirium rating scale; CGI-S: Clinical Global Impression-Severity scale; MDAS; Memorial Assessment Delirium Scale; KPS: Karnofsky Performance Status scale; ICU-DSC: Delirium Screening Checklist; DI: Delirium Index; RC: Ross Chouinard scale; SAs: Simpson and Angus Scale; DRS-R-98: Delirium rating scale revised 98; DSM-IV: Diagnostic and Statistical Manual of Mental Disorders $4^{\text {th }}$ edition.

ISSN: 1659-2441 Revista electrónica publicada por el Departamento de Farmacología de la Escuela de Medicina de la Universidad de Costa Rica, 2060 San José, Costa Rica. ${ }^{\circledR}$ All rights reserved. 